\title{
Revisiting electroweak phase transition with varying Yukawa coupling constants
}

\author{
Arianna Braconi, ${ }^{*}$ Mu-Chun Chen, ${ }^{\dagger}$ and Geoffrey Gaswint ${ }^{\ddagger}$ \\ Department of Physics and Astronomy, University of California, Irvine, California 92697-4575, USA
}

(Received 22 January 2019; published 19 July 2019)

\begin{abstract}
We revisited the scenario of electroweak baryogenesis in the presence of large Yukawa couplings, in which it was previously found that a strongly first-order electroweak phase transition can occur. Given the sensitivity of the running of the Higgs quartic coupling on the Yukawa couplings, we find that large Yukawa couplings drastically lower the scale at which the Higgs potential becomes unstable. Specifically, with only two quarks having order one Yukawa coupling, the scalar potential becomes unstable at the TeV scale, assuming the standard model values for the Higgs sector parameters at the electroweak scale. Furthermore, if assuming the standard model values for the Higgs sector parameters at the TeV scale, the Higgs quartic coupling constant becomes too large at the electroweak scale. This in turn predicts a much lighter Higgs mass than the measured value of $125 \mathrm{GeV}$. The strength of the electroweak phase transition is also significantly weakened in this scenario.
\end{abstract}

DOI: $10.1103 /$ PhysRevD.100.015032

\section{INTRODUCTION}

The origin of the observed cosmological matter-antimatter asymmetry of the Universe remains an outstanding question in both particle physics and cosmology. Sakharov pointed out [1] that in order for the matter-antimatter asymmetry to be generated dynamically, three conditions are required: (i) baryon number violation, (ii) $\mathrm{C}$ and $C P$ violations, and (iii) departure from thermal equilibrium. There are three possible ways to realize departure from thermal equilibrium that have been utilized in mechanisms for baryogenesis: (i) out-of-equilibrium decay of heavy particles, (ii) electroweak phase transition, and (iii) dynamics of topological defects.

In electroweak baryogenesis (EWBG), the out-ofequilibrium condition is achieved if the electroweak phase transition (EWPT) is strongly first order. This in turn requires a light Higgs mass, $m_{H} \lesssim 72 \mathrm{GeV}$ [2], in the standard model. Clearly this constraint is in conflict with the observed Higgs mass of $125 \mathrm{GeV}$. In the constrained minimal supersymmetric standard model, a strongly firstorder EWPT also requires a light Higgs boson which is inconsistent with observation. However, it has been shown

\footnotetext{
*abraconi@uci.edu

†muchunc@uci.edu

ggaswint@uci.edu
}

Published by the American Physical Society under the terms of the Creative Commons Attribution 4.0 International license. Further distribution of this work must maintain attribution to the author(s) and the published article's title, journal citation, and DOI. Funded by SCOAP. that a very narrow parameter space can be made consistent with the observed Higgs mass and the strong first-order phase transition, by fine-tuning the supersymmetric parameters (see, for example, [3]).

Several approaches have been proposed to obtain a strongly first-order EWPT while maintaining a Higgs mass consistent with observation. One way is to expand the scalar sector by adding scalar singlet(s) [4] or by adding electroweak triplet scalar fields [5]. An interesting explanation for EWBG via a two Higgs doublet model and heavy top quarks with large Yukawa couplings was presented in [6]. This allows for a first-order phase transition and EWBG. This scenario does not suffer from the problems to be discussed here, because the heavy quarks only couple to the second Higgs doublet and therefore do not impact substantially the renormalization group equations (RGEs) of the standard model Higgs parameters. This scenario will have phenomenological implications that can be tested for at the LHC.

It was originally pointed out in [7] that additional large Yukawa coupling constants in the early Universe can drive the electroweak phase transition to be strongly first order. The Frogatt-Nielsen mechanism is proposed for driving the variation in the Yukawa couplings, making them large before and during the EWPT. This paper [7] focused on generating enough $C P$ violation to explain the baryon asymmetry of the Universe, and concluded that the scalar sector can be extended to obtain a first-order EWPT. In [8] a model-independent calculation was done to demonstrate that a first-order electroweak phase transition can be obtained from large Yuakawa couplings that depend explicitly on the Higgs vacuum expectation value (vev). 
This was then taken further in [9] where the FroggattNielsen mechanism was implemented, so that the Yukawa couplings depend on the vev of a new scalar field.

Large Yukawa couplings will also have a substantial effect on the running of the Higgs quartic coupling, given that the $\beta$ function for the quartic coupling is sensitive to changes in the Yukawa couplings. When the Higgs sector parameters are kept at their standard model values at the weak scale, increasing the Yukawa couplings to be of order 1 lowers the scale at which the quartic coupling becomes negative, thus lowering the scale at which the Higgs potential becomes unstable. If the value of the quartic coupling in the UV is kept at the standard model value, the renormalization group corrections driven by large Yukawa couplings then lead to a quartic coupling value that is larger than the standard model value at the weak scale. Consequently, this results in a light Higgs mass that is in conflict with observation.

The paper is organized as follows: in Sec. II, we review the calculation of the effective scalar potential at finite temperature. This is then followed by Sec. III where we study the renormalization group evolution of the coupling constants and the stability of the scalar potential. In Sec. IV, we present the analysis of the electroweak phase transition by including the effects of the renormalization group corrections. Section V concludes the paper.

\section{REVIEW OF EFFECTIVE POTENTIAL CALCULATION}

The calculation of the effective potential was developed $[10,11]$ using the path integral formalism. For a review, see [12]. The effective scalar potential is given by the tree-level potential, plus the one-loop zero-temperature correction, the one-loop finite-temperature correction, and the daisy correction.

$$
\begin{aligned}
V_{\text {eff }}(\phi, T)= & V_{0}(\phi)+V_{1}^{T=0}(\phi)+V_{1}^{T \neq 0}(\phi, T) \\
& +V_{\text {Daisy }}(\phi, T)
\end{aligned}
$$

Both the zero-temperature and finite-temperature one-loop corrections receive contributions from all particles coupled to the Higgs, while the daisy correction receives contributions only from bosons.

In [9], an analysis of the effective potential and phase transition with varying Yukawa couplings was carried out. The Yukawa couplings are taken to be order 1 before the electroweak phase transition, then assume their observed standard model values after the phase transition. The ansatz to account for this variation in the Yukawa couplings was given as

$$
y(\phi)=\left\{\begin{array}{ll}
y_{1}\left(1-\frac{\phi}{v}\right)+y_{0} & 0 \leq \phi \leq v \\
y_{0} & v \leq \phi
\end{array},\right.
$$

where $\phi$ is the value of the Higgs field, and $y_{1}$ and $y_{0}$ are constants. The same ansatz is used in this analysis.

In this case, the mass of the fermions would also depend on the Higgs vev through $y(\phi)$, and can take the form

$$
m_{f}(\phi)=\frac{y(\phi) \phi}{\sqrt{2}} .
$$

If additional scalars are added, then the mass of the fermions can also be dependent on powers of the vev of those scalars, as dictated by the Frogatt-Nielsen mechanism.

The tree-level potential, $V_{0}$, used in this calculation is given by

$$
V_{0}=-\mu^{2}|H|^{2}+\lambda|H|^{4},
$$

with $\mu^{2}>0$ and electroweak symmetry is broken by the usual Higgs doublet

$$
H=\left(0, \frac{\phi}{\sqrt{2}}\right)
$$

The one-loop contribution at zero temperature is obtained by summing over all 1PI diagrams with one loop and zero external momenta. After renormalizing using the $\overline{\mathrm{MS}}$ scheme, the one-loop zero-temperature contribution is given by

$$
\begin{aligned}
V_{1}^{T=0}(\phi)= & \sum_{i} \frac{n_{i}(-1)^{F_{i}}}{64 \pi^{2}}\left[m_{i}^{4}(\phi)\left(\log \left[\frac{m_{i}^{2}(\phi)}{m_{i}^{2}(v)}\right]-\frac{3}{2}\right)\right. \\
& \left.+2 m_{i}^{2}(\phi) m_{i}^{2}(v)\right]
\end{aligned}
$$

where the sum is over all particles that couple to the Higgs. $F$ is 0 or 1 for bosons and fermions, respectively, and $n_{i}$ is the particle's multiplicity ( 1 for each boson, 4 for colorless Dirac fermions, and 12 if the fermion also carries color). $V_{1}^{T=0}(\phi)$ already includes all counterterms necessary to maintain the tree-level values of the mass and coupling constant.

To calculate the finite-temperature contributions, it is necessary to use the finite-temperature Feynman rules and to sum over the Matsubara frequencies. The result is given by

$$
V_{1}^{T \neq 0}(\phi, T)=\sum_{i} \frac{n_{i}(-1)^{F_{i}} T^{4}}{2 \pi^{2}} J_{\mathrm{B} / \mathrm{F}}\left(\frac{m(\phi)^{2}}{T^{2}}\right),
$$

where $J_{B / F}$ is the thermal bosonic/fermionic function

$$
J_{B / F}=\int_{0}^{\infty} d x x^{2} \log \left[1-(-1)^{F} e^{-\sqrt{x^{2}+m^{2} / T^{2}}}\right] .
$$


This integral has convenient high-temperature approximations for $m^{2} / T^{2} \ll 1$,

$$
\begin{gathered}
J_{\mathrm{B}}\left(\frac{m^{2}}{T^{2}}\right)=-\frac{\pi^{4}}{45}+\frac{\pi^{2}}{12} \frac{m^{2}}{T^{2}}-\frac{\pi}{6}\left(\frac{m^{2}}{T^{2}}\right)^{3 / 2} \\
-\frac{1}{32} \frac{m^{4}}{T^{4}} \log \left(\frac{m^{2}}{a_{b} T^{2}}\right), \\
J_{\mathrm{F}}\left(\frac{m^{2}}{T^{2}}\right)=\frac{7 \pi^{4}}{360}-\frac{\pi^{2}}{24} \frac{m^{2}}{T^{2}}-\frac{1}{32} \frac{m^{4}}{T^{4}} \log \left(\frac{m^{2}}{a_{f} T^{2}}\right),
\end{gathered}
$$

where the mass dependence on $\phi$ is understood. In this analysis the thermal bosonic and fermionic functions were evaluated numerically, since near the critical temperature the condition that $m^{2} / T^{2} \ll 1$ is violated. As observed in [9], if varying Yukawa couplings are assumed, the mass squared term in the fermion contribution can produce a cubic term in $\phi$, normally only present in the bosonic contribution. This has the effect of creating a barrier in the potential, thus strengthening the phase transition and allowing for a first-order phase transition.

The one-loop approximation is valid at temperatures below the critical temperature; however, near the critical temperature this approximation breaks down. Quadratically divergent bubbles that add a factor of $\lambda T^{2} / \mathrm{m}^{2}$ to the twopoint functions can be safely ignored at low temperatures where $\lambda T^{2} / m^{2} \ll 1$. At and above the critical temperature, these diagrams (called daisy, or ring diagrams) must be accounted for by resumming over an infinite number of diagrams at every order. This is equivalent to replacing the particle mass by an effective mass, $m^{2} \rightarrow m^{2}+\Pi(T)$, where $\Pi(T)$ is the thermal mass of the particle. The daisy contribution is given by

$$
V_{\text {Daisy }}(\phi, T)=\sum_{i} \frac{n_{i} T}{12 \pi}\left(m_{i}^{3}(\phi)-\left[m_{i}^{2}(\phi)+\Pi_{i}(T)\right]^{3 / 2}\right) .
$$

In the case of the Higgs (with constant Yukawa couplings) the thermal mass calculated in [13] is used,

$$
\Pi_{\phi}(T)=\left(\frac{3}{16} g_{2}^{2}+\frac{1}{16} g_{y}^{2}+\frac{\lambda}{2}+\frac{y_{t}^{2}}{4}\right) T^{2} .
$$

And with varying Yukawa couplings,

$$
\Pi_{\phi}(\phi, T)=\left(\frac{3}{16} g_{2}^{2}+\frac{1}{16} g_{y}^{2}+\frac{\lambda}{2}+\frac{y_{t}^{2}}{4}+\frac{n_{*} y(\phi)^{2}}{48}\right) T^{2} .
$$

Here $n_{*}$ is taken to be 60 to account for the additional five quarks; $g_{2}$ and $g_{y}$ are the weak and hypercharge coupling constants.
There are three effects of the large Yukawa couplings during the electroweak phase transition: (i) In the $T=0$ one-loop corrections, the large Yukawa couplings result in the lowering of the scalar potential in the region of $0<\langle\phi\rangle<v$ thus weakening the phase transition; (ii) the finite temperature one-loop correction from the fermions adds to the potential and strengthens the phase transition; (iii) the large Yukawa coupling present in the Higgs daisy correction also strengthens the phase transition. However, this does not take into account the effects the large Yukawa couplings will have on the RGEs for the Higgs sector parameters.

\section{STABILITY OF THEORY WITH ADDITIONAL YUKAWA COUPLINGS OF ORDER UNITY}

In the standard model, the Higgs quartic coupling becomes negative around $10^{10} \mathrm{GeV}$, rendering the Higgs potential unstable. Increasing the number of Yukawa couplings that are of order 1 drastically lowers the scale at which the quartic coupling becomes negative. This can be seen analytically from the one-loop $\beta$ function [Eq. (A3) in Appendix A]. If we assume only one large quark Yukawa coupling and no CKM mixing, then the leading order terms can be approximated as

$$
\beta_{\lambda}^{(1)} \approx 24 \lambda^{2}+12 \lambda y^{2}-6 y^{4} .
$$

If additional quark Yukawa couplings of order 1 are added, the $\beta$ function becomes

$$
\beta_{\lambda}^{(1)} \approx 24 \lambda^{2}+12 \lambda n y^{2}-6 n y^{4},
$$

where $n$ is the number of order one Yukawa couplings. The addition of these large Yukawa couplings makes the negative term dominant, driving the Higgs quartic coupling negative at a lower scale. Here, the effects of the large Yukawa couplings on the evolution of $\beta_{\lambda}^{(1)}$ are independent of the specific variation used in the Yukawa couplings, and can include a broad range of models where the additional scalar fields are heavy. In the case of additional light scalars, the beta functions need to be calculated (dependent on the model) to incorporate those effects.

To evaluate the full RGEs with large Yukawa couplings, and their effect on the running of the Higgs quartic coupling, the Mathematica package SARAH [14] was used. In Fig. 1, the number of Yukawa couplings equal to 1.0 were successively increased at the electroweak scale. The top quark Yukawa coupling and all other parameters were kept at their standard model values. It is clear that this drastically lowers the scale at which the Higgs quartic coupling becomes negative, pushing it very close to the electroweak scale.

In the next case considered, the order one Yukawa couplings are imposed at $1 \mathrm{TeV}$. Figure 2(a) has Yukawa 


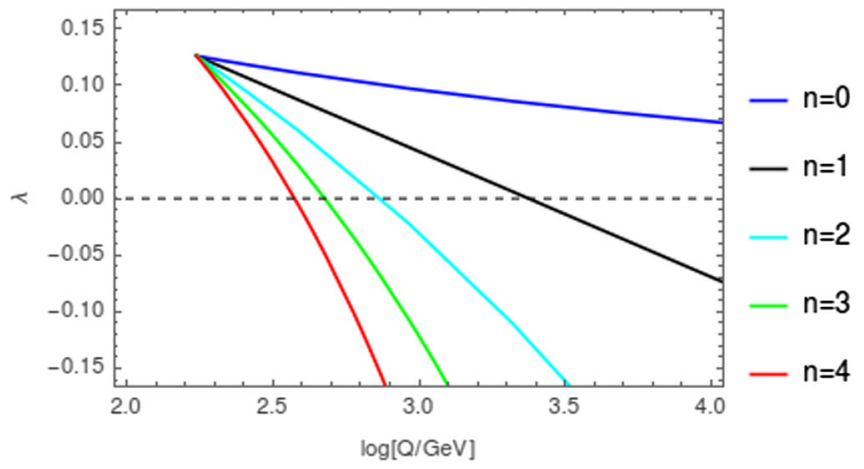

FIG. 1. RGE running of the Higgs quartic coupling with additional Yukawa couplings equal to 1.0, and standard model boundary conditions imposed at the electroweak scale. The number of additional Yukawa couplings is given by $n$, with $n=0$ corresponding to the case of the standard model.

couplings equal to 1.0, while Fig. 2(b) has Yukawa couplings equal to 2.0. As shown in the figures, setting the Yukawa couplings to be order 1 or greater increases the Higgs quartic coupling at the electroweak scale. This in turn has the effect of lowering the Higgs mass, as seen in the following section.

\section{RENORMALIZATION GROUP IMPROVED EFFECTIVE POTENTIAL AND ELECTROWEAK PHASE TRANSITION}

At the electroweak scale, the parameters of the Higgs potential are $\mu=89$ and $\lambda=0.13$. This allows for a firstorder phase transition with varying Yukawa couplings at $115 \mathrm{GeV}$, and shown by the solid blue line in Fig. 3(a). However, this does not take into account the effects that additional large Yukawa couplings would have on the running of the RGEs and therefore on the values of the Higgs parameters at that scale.

To account for these effects, the RGEs were run downwards from the $\mathrm{TeV}$ scale, with a successively increased number of large Yukawa couplings. The potential of the two most extreme cases are shown, where the top Yukawa coupling retains its standard model value but the five additional quark Yukawa couplings are set equal to 1.0 and 2.0, corresponding to the orange and green curves in Figs. 3(a) and 3(b).

The large Yukawa couplings increase the value of the quartic coupling at the electroweak scale, which in turn lowers both the vev and the temperature at which a phase transition occurs. For a transition to be considered strongly

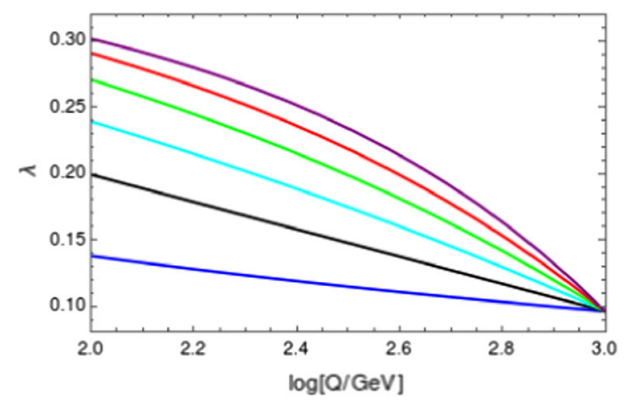

(a) Additional Yukawa couplings equal to 1.0

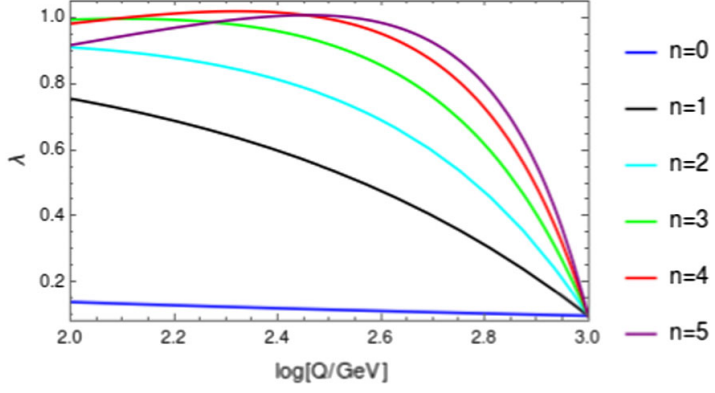

(b) Additional Yukawa couplings equal to 2.0

FIG. 2. RGE running of the Higgs quartic coupling with additional Yukawa couplings of order 1 and boundary conditions imposed at the TeV scale. The number of additional Yukawa couplings is given by $n ; n=0$ corresponds to the case of the standard model.

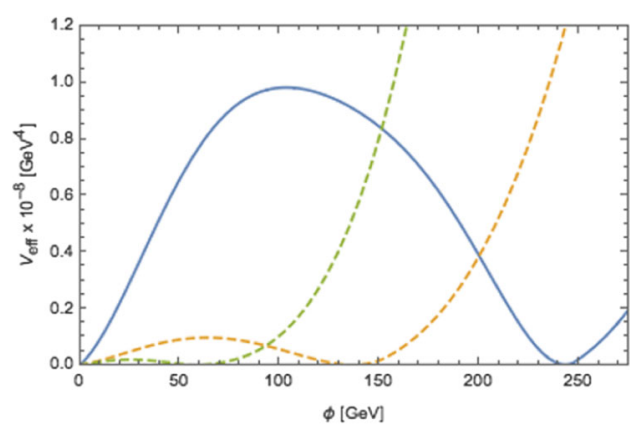

(a) All effective potentials

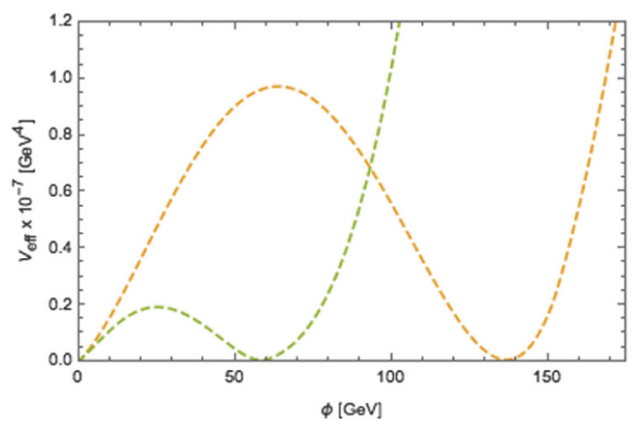

(b) RGE-improved effective potentials only

FIG. 3. The effective potential with varying Yukawa couplings. Solid blue line: The effective potential with $y_{1}=2.0$, and standard model values of the Higgs sector parameters. Dashed lines: The effective potential with RGE-improved values of the Higgs sector parameters. The orange curve uses $y_{1}=1.0$; the green curve uses $y_{1}=2.0$. 
first order, it must meet the condition that $\phi_{c} / T_{c} \gtrsim 1$ [15]. In the case of the additional five quark Yukawa couplings equal to 1.0, shown by the orange curve in Fig. 3(b), the quartic coupling increases to 0.27 , which lowers the vev to $137 \mathrm{GeV}$, leading to a Higgs mass of $95 \mathrm{GeV}$. In this scenario the critical temperature decreases to $112 \mathrm{GeV}$, and $\phi_{c} / T_{c}$ decreases to 1.23. In the case of additional Yukawa couplings equal to 2.0, shown by the green curve in Fig. 3(b), the quartic coupling increases to 0.97, the vev decreases to only $58 \mathrm{GeV}$, and the critical temperature decreases to $52 \mathrm{GeV}$, with $\phi_{c} / T_{c}=1.12$. The predicted Higgs mass in this case is also lowered, to $80 \mathrm{GeV}$.

\section{CONCLUSION}

Requiring that the electroweak phase transition in the standard model be strongly first order constrains the Higgs mass to be $m_{H} \lesssim 72 \mathrm{GeV}$. With an observed Higgs mass of $125 \mathrm{GeV}$, it is clear that a strongly first order electroweak phase transition requires physics beyond the standard model. A new scenario was proposed in Refs. $[7,8]$ which introduces the idea that large Yukawa couplings could cause a strongly first-order electroweak phase transition.

The Higgs quartic coupling in the standard model is positive up to around $10^{10} \mathrm{GeV}$, where the quartic coupling then turns negative, making the Higgs scalar potential unstable. If additional Yukawa couplings of order 1 are present before the electroweak phase transition, the Higgs potential becomes unstable at a much lower scale. If the large Yukawa couplings are present at the $\mathrm{TeV}$ scale, the Higgs quartic coupling is driven to be larger at the electroweak scale than its standard model value. This in turn predicts a lighter Higgs than the measured value. In addition to the inconsistency with the observed Higgs mass, there also exist severe constraints [16] from cosmology on the proposed scenario with varying Yukawa couplings. All together, these limitations render this simplest setup with large varying Yukawa couplings not a viable mechanism for baryogenesis.

\section{ACKNOWLEDGMENTS}

We thank Michael Ratz for posing the question which led to the project and for discussions. We also thank Iason Baldes for helpful communications. The work of A. B. is supported, in part, by a GAANN fellowship. The work of M.-C. C. is supported, in part, by the National Science Foundation under Grant No. PHY-1620638. M.-C. C. thanks the hospitality of the Aspen Center for Physics, which is supported by National Science Foundation Grant No. PHY-1607611, where part of this work was performed.

\section{APPENDIX: RENORMALIZATION GROUP EQUATIONS}

Appendix A shows the RGEs at one loop that are used in our analysis. The RGEs for the Higgs quartic coupling, and scalar mass at one loop for the standard model [17], are

$$
\begin{aligned}
& \frac{d \lambda}{d t}=\frac{1}{16 \pi^{2}} \beta_{\lambda}^{(1)}, \\
& \frac{d \mu}{d t}=\frac{1}{16 \pi^{2}} \beta_{\mu}^{(1)},
\end{aligned}
$$

where $t=\ln E$, where $\mathrm{E}$ is the running scale. The betafunction coefficients are given by

$$
\begin{aligned}
\beta_{\lambda}^{(1)}= & 24 \lambda^{2}-\frac{9}{5} g_{1}^{2} \lambda-9 g_{2}^{2} \lambda+\frac{9}{8}\left(\frac{3}{25} g_{1}^{4}+\frac{2}{5} g_{1}^{2} g_{2}^{2}+g_{2}^{4}\right) \\
& +4 \lambda \operatorname{Tr}\left(3 Y_{u}^{\dagger} Y_{u}+3 Y_{d}^{\dagger} Y_{d}+Y_{e}^{\dagger} Y_{e}\right) \\
& -2 \operatorname{Tr}\left(3\left(Y_{u}^{\dagger} Y_{u}\right)^{2}+3\left(Y_{d}^{\dagger} Y_{d}\right)^{2}+\left(Y_{e}^{\dagger} Y_{e}\right)^{2}\right), \\
\beta_{\mu}^{(1)}= & 12 \mu \lambda+2 \mu \operatorname{Tr}\left(Y_{e} Y_{e}^{\dagger}\right)+6 \mu \operatorname{Tr}\left(Y_{d} Y_{d}^{\dagger}\right)+6 \mu \operatorname{Tr}\left(Y_{u} Y_{u}^{\dagger}\right) \\
& -\frac{9}{10} g_{1}^{2} \mu-\frac{9}{2} g_{2}^{2} \mu .
\end{aligned}
$$

The gauge couplings for the standard model at the one-loop level are

$$
\frac{d g_{i}}{d t}=\frac{1}{16 \pi^{2}} \beta_{g_{i}}^{(1)}
$$

where the three $\beta_{g_{i}}$ functions are expressed as

$$
\begin{aligned}
& \beta_{g_{1}}^{(1)}=\frac{41}{10} g_{1}^{3}, \\
& \beta_{g_{2}}^{(1)}=-\frac{19}{6} g_{2}^{3}, \\
& \beta_{g_{3}}^{(1)}=-7 g_{3}^{3} .
\end{aligned}
$$

The RGEs for the Yukawa couplings in the standard model at one loop are summarized as

$$
\begin{aligned}
\beta_{Y_{u}}^{(1)}= & -\frac{3}{2}\left(-Y_{u} Y_{u}^{\dagger} Y_{u}+Y_{u} Y_{d}^{\dagger} Y_{d}\right) \\
& +Y_{u}\left(3 \operatorname{Tr}\left(Y_{d} Y_{d}^{\dagger}\right)+3 \operatorname{Tr}\left(Y_{u} Y_{u}^{\dagger}\right)\right. \\
& \left.-8 g_{3}^{2}-\frac{17}{20} g_{1}^{2}-\frac{9}{4} g_{2}^{2}+\operatorname{Tr}\left(Y_{e} Y_{e}^{\dagger}\right)\right),
\end{aligned}
$$

$$
\begin{aligned}
\beta_{Y_{d}}^{(1)}= & \frac{1}{4}\left(6\left(-Y_{d} Y_{u}^{\dagger} Y_{u}+Y_{d} Y_{d}^{\dagger} Y_{d}\right)-Y_{d}\left(-12 \operatorname{Tr}\left(Y_{d} Y_{d}^{\dagger}\right)\right.\right. \\
& \left.\left.-12 \operatorname{Tr}\left(Y_{u} Y_{u}^{\dagger}\right)+32 g_{3}^{2}-4 \operatorname{Tr}\left(Y_{e} Y_{e}^{\dagger}\right)+9 g_{2}^{2}+g_{1}^{2}\right)\right),
\end{aligned}
$$

$$
\begin{aligned}
\beta_{Y_{e}}^{(1)}= & \frac{3}{2} Y_{e} Y_{e}^{\dagger} Y_{e}+Y_{e}\left(3 \operatorname{Tr}\left(Y_{d} Y_{d}^{\dagger}\right)+3 \operatorname{Tr}\left(Y_{u} Y_{u}^{\dagger}\right)\right. \\
& \left.-\frac{9}{4} g_{1}^{2}-\frac{9}{4} g_{2}^{2}+\operatorname{Tr}\left(Y_{e} Y_{e}^{\dagger}\right)\right) .
\end{aligned}
$$


[1] A. D. Sakharov, Pis'ma Zh. Eksp. Teor. Fiz. 5, 32 (1967); Usp. Fiz. Nauk 161, 61 (1991).

[2] C. Grojean, G. Servant, and J. D. Wells, Phys. Rev. D 71, 036001 (2005).

[3] M. Carena, G. Nardini, M. Quiros, and C. E. M. Wagner, J. High Energy Phys. 02 (2013) 001.

[4] J. R. Espinosa and M. Quiros, Phys. Rev. D 76, 076004 (2007).

[5] M. Garcia-Pepin and M. Quiros, J. High Energy Phys. 05 (2016) 177.

[6] K. Fuyuto, W.-S. Hou, and E. Senaha, Phys. Lett. B 776, 402 (2018).

[7] M. Berkooz, Y. Nir, and T. Volansky, Phys. Rev. Lett. 93, 051301 (2004).

[8] I. Baldes, T. Konstandin, and G. Servant, J. High Energy Phys. 12 (2016) 073.
[9] I. Baldes, T. Konstandin, and G. Servant, Phys. Lett. B 786, 373 (2018).

[10] S. R. Coleman and E. J. Weinberg, Phys. Rev. D 7, 1888 (1973).

[11] R. Jackiw, Phys. Rev. D 9, 1686 (1974).

[12] M. Quiros, in Proceedings of the Summer School in Highenergy physics and cosmology: Trieste, Italy, 1998 (1999), pp. 187-259 [arXiv:hep-ph/9901312].

[13] A. Katz and M. Perelstein, J. High Energy Phys. 07 (2014) 108.

[14] F. Staub, Adv. High Energy Phys. 2015, 840780 (2015).

[15] A. Ahriche, Phys. Rev. D 75, 083522 (2007).

[16] B. Lillard, M. Ratz, M. P. Tait, Tim, and S. Trojanowski, J. Cosmol. Astropart. Phys. 07 (2018) 056.

[17] H. Arason, D. J. Castano, B. Keszthelyi, S. Mikaelian, E. J. Piard, P. Ramond, and B. D. Wright, Phys. Rev. D 46, 3945 (1992). 